Published in final edited form as:

Curr Oncol Rep. ; 20(10): 80. doi:10.1007/s11912-018-0729-3.

\title{
The Role of Oncolytic Viruses in the Treatment of Melanoma
}

Claire-Audrey Y. Bayan, BA ${ }^{a}$, Adriana T. Lopez, BA ${ }^{a}$, Robyn D. Gartrell, MD ${ }^{b}$, Kimberly M. Komatsubara, MD $^{\mathrm{b}}$, Margaret Bogardus, BA $^{\mathrm{a}}$, Nisha Rao, MD $^{\mathrm{b}}$, Cynthia Chen, BS $^{\mathrm{a}}$, Thomas D. Hart, BA ${ }^{\mathrm{e}}$, Thomas Enzler, MD $^{\mathrm{b}}$, Emanuelle M. Rizk, BA ${ }^{\mathrm{b}}$, Jaya Sarin Pradhan, DMD, MPH $^{d}$, Douglas K. Marks, MD $^{b}$, Larisa J. Geskin, MD $^{c}$, and Yvonne M. Saenger, MD $^{b}$ ${ }^{a}$ Columbia University College of Physicians and Surgeons, Columbia University Medical Center, New York, NY 10032. (Tel: 212-305-2862)

bepartment of Medicine, Division of Hematology/Oncology, Columbia University Medical Center, New York, NY 10032. (Tel: 212-305-0455)

'Department of Dermatology, Columbia University Medical Center, New York, NY 10032. (Tel: 212-305-5293)

dDepartment of Pathology; Columbia University Medical Center, New York, NY 10032. (Tel: 212-305-6719)

eColumbia University, Columbia College, New York, NY 10027. (Tel: 619-866-8058)

\section{Abstract}

Purpose of review: Oncolytic virotherapy is a new approach to the treatment of cancer and its success in the treatment of melanoma represents a breakthrough in cancer therapeutics. This paper provides a review of the current literature on the use of oncolytic viruses (OVs) in the treatment of melanoma.

Recent findings: T-VEC is the first OV approved for the treatment of melanoma and presents new challenges as it enters the clinical setting. Several other OVs are at various stages of clinical and pre-clinical development for the treatment of melanoma. Reports from phase Ib-III clinical trials combining T-VEC with checkpoint blockade are encouraging and demonstrate potential added benefit of combination immunotherapy.

Summary: OVs have recently emerged as a standard treatment option for patients with advanced melanoma. Several OVs and therapeutic combinations are in development. Immuno-oncolytic virotherapy combined with immune checkpoint inhibitors is promising for the treatment of advanced melanoma.

\section{Keywords}

Oncolytic virus; Oncolytic viral therapy; Melanoma; Immunotherapy; Talimogene Laherparepvec (T-VEC); Tumor microenvironment; Biosafety; Combination Therapy

Corresponding Author: Yvonne Saenger, Address: 161 Fort Washington Avenue, New York, NY 100232, Phone: 212-305-0455, Fax:, yms4@cumc.columbia.edu.

Conflicts of Interest: none 


\section{Introduction}

Oncolytic viruses (OVs) are native or modified viruses that selectively infect malignant cells and induce direct tumor cell lysis as well as potential systemic anti-tumor response. Until recently, limitations related to severe toxicities associated with viral infection, tumor cell selectivity and escape, and inadequate oncolytic potency made oncolytic virotherapy impractical and impeded further development.

The modern era of oncolytic virotherapy began in the early 1990s with a landmark study in which a mutant herpes virus lacking thymidine kinase was observed to successfully cause lysis of glioma tumor cells both in vitro and in vivo. $(1,2)$ Over the past several years, there has been an intensification of laboratory, pre-clinical, and clinical research in the field of oncolytic virotherapy, culminating in over 40 clinical trials involving OVs recruiting patients in 2017.

Oncolytic virotherapy is a novel treatment modality for advanced melanoma. Currently, Talimogene laherparepvec (T-VEC) is the only OV that is approved by the U.S. Food and Drug Administration (FDA) for the treatment of cancer, specifically advanced melanoma. Other oncolytic viruses have been investigated as monotherapy and in combination for the treatment of melanoma including herpes simplex virus (HSV), HF-10 (HF-10), coxsackieviruses, reoviruses, and poxvirus, and will be discussed further below. The current limitations and challenges facing the treatment of melanoma with OVs will also be reviewed.

\section{Mechanism of Action}

The goal of OV delivery is to generate strong local and systemic immune responses against tumor cells while minimizing collateral effects of immune stimulation including rapid inactivation of the therapeutic virus, as well as viral and immune toxicity to normal cells.(3) OV design and administration thus reflect these therapeutic goals. OVs are modified or selected to preferentially infect tumor cells.(4) Several OVs preferentially bind to entry receptors disproportionately exhibited on tumor cells, increasing specificity for tumor cell infectivity.(5) Viral entry receptors can also be engineered to target tumor cells.(6) Local injection of the $\mathrm{OV}$ allows for concentration of the virus at the site of the tumor.

Immunosuppressed tumor microenvironments, such as in melanoma, are ideal for viral replication.(7) With lymphatics and vasculature frequently dysregulated in cancer, this serves to shield the virus from attack by the innate immune response.(8) Within this environment, viruses then exploit tumor cell survival mechanisms such as hyperactive cell replication machinery, unresponsiveness to interferon signaling, and inhibition of apoptosis. (9) Specificity for tumor can then be enhanced by engineering viruses to make cancerspecific proteins required for replication.(10)

Viral replication ultimately leads to tumor cell death by necrosis or pyroptosis, resulting in release of tumor antigens, DAMPs, viral PAMPs, and cytokines.(11) This triggers maturation of antigen presenting cells which traffic to regional lymph nodes and stimulate the proliferation of tumor antigen-specific $\mathrm{T}$ cells. This ultimately results in the targeted 
killing of infected and uninfected tumor cells by activated adaptive and innate immune cells. (11-13) Recruitment of innate and adaptive immunity allows for a systemic response to tumor cells with the potential to cause regression of tumor at non-injected sites.(14) Therefore, OV tumor cell infection can transform the tumor microenvironment (TME) from immune-suppressed to immune-activated.(15, 16)

OVs can be armed with a variety of genes to increase viral efficacy and immunogenicity of tumor cell death. For example, matrix-degrading enzymes can improve intratumoral viral dissemination.(17) Fusogenic membrane glycoproteins can promote syncitia formation, allowing viruses to spread between cells without immune detection until cell lysis.(18) Granulocyte-macrophage colony stimulating factor (GM-CSF) can improve trafficking of antigen-presenting cells to lymph nodes,(19) and chemokines can improve T cell infiltration into the tumor milieu.(20) Finally, prodrug convertase enzymes can facilitate conversion of infused anti-cancer drugs to active forms.(21)

\section{Oncolytic Viruses Used in the Treatment of Melanoma}

T-VEC is currently the only OV approved in the U.S. and Europe for the treatment of advanced melanoma. There is a significant amount of clinical research underway focused on the use of OVs in the treatment of melanoma (Table 1.) Combination therapy is also an active area of research (Table 2). Given the immunosuppressed nature of the TME in melanoma, combination therapy with OVs has been proposed as a means to generate a proinflammatory milieu and prime anti-tumor immunity. (22) Combinations include pairing OVs with various modalities and agents such as radiation therapy, radionuclide therapy, chemotherapy, biologic therapies, and other viruses, and provide additional venues for investigation in melanoma.(23) Results from phase Ib-II trials combining OVs with immune checkpoint inhibitors are promising and have reported overall good treatment tolerance and effect, and phase III trials are ongoing.

\section{Oncolytic Herpes Simplex Viruses}

Talimogene laherparepvec (T-VEC) - T-VEC is a first-in-class recombinant HSV (type 1) that has been engineered to contain mutations in the infectious cell proteins (ICP) 34.5 and 47 and expresses GM-CSF. ICP34.5 is involved in viral replication, viral exit from cells, and inhibition of the host's protein synthesis shut-off response, which is normally activated upon viral infection. Mutations in ICP34.5 protect normal cells from HSV replication and selectively target cancer cells, which are deficient in the protein synthesis shut-off response due to deficiencies in cellular growth arrest and DNA damage-inducible protein 34 (GADD34 or MyD116).(24-26) ICP47 inhibits transporter associated with antigen presentation (TAP), a protein involved in the transfer of major histocompatibility complex class I restricted antigens from the cytosol to the plasma membrane.(27, 28) Thus, mutations in ICP34.5 and ICP47 in the HSV gene collectively result in selective replication of the virus in tumor cells and enhanced antigen presentation of HSV-infected cells. GM-CSF has been demonstrated to augment the anti-tumor immune response and induce a systemic immune response.(29) 
In the phase I study evaluating T-VEC in multiple solid tumor types, the most commonly observed adverse events were flu-like symptoms and local injection site reactions, with more marked side effects noted in patients who were seronegative for HSV.(30) Subsequently, a phase II study evaluating T-VEC in patients with unresectable stage IIIc-IV melanoma with injectable lesions was conducted.(31) A total of 50 patients were enrolled with an overall response rate (ORR) of 26\%, including 8 complete responses (CR) and 5 partial responses (PR). The overall survival was $58 \%$ at 1 year and $52 \%$ at 2 years. Responses were observed in both injected and distant lesions. In addition, both local and systemic immune responses were demonstrated by an increase in melanoma antigen recognized by T cells 1 (MART-1) specific $\mathrm{T}$ cells locally and systemically, as well as a decrease in regulatory $\mathrm{T}$ cells and myeloid-derived suppressive cells (MDSC) in the injected regressing lesions.(32)

Based on the data from phase I/II trials, a phase III study (OPTiM) was conducted.(33) This study randomized 436 patients with unresectable and injectable stage IIIB-IV melanoma to receive treatment with either intralesional T-VEC or GM-CSF. This study met its primary endpoint of durable response rate (DRR) (defined as a continuous response lasting $\geq 6$ months), with a significantly higher DRR observed in the T-VEC-treated group compared to the GM-CSF-treated group (16.3\% v. $2.1 \%$; odds ratio, 8.9 ; $p<0.001)$. Notably, responses were observed in $15 \%$ of uninjected visceral metastases, suggesting a systemic immune effect. There was a trend toward improved overall survival in the T-VEC treated group (23.3 months in TVEC group versus 19.9 months in GM-CSF group; HR, 0.79; $\mathrm{p}=0.051$ ). In a subset analysis, highly significant treatment benefit was found in patients with stage IIIB, IIIC, or IVM1a disease and treatment-naïve patients, and no difference was observed based on HSV serological status. As a result of this study, T-VEC was approved by the FDA in October 2015 and the European Medicines Agency in December 2015 for the treatment of unresectable stage III and IV melanoma.

Given the evidence suggesting generation of a systemic immune response by T-VEC, combination therapy with checkpoint inhibitors are of particular interest. In a phase Ib trial, T-VEC plus ipilimumab was evaluated in 18 patients with advanced melanoma.(34) The ORR was $50 \%$ (complete remission $(\mathrm{CR})=22 \%$; partial response $(\mathrm{PR})=28 \%$; stable disease $(\mathrm{SD})=22 \%)$ and overall survival $(\mathrm{OS})$ was $67 \%$ with 18 -month progression-free survival (PFS) at $50 \%$. Eight patients (44\%) had a durable response of $\geq 6$ months. Of reported adverse events (AEs), $26.3 \%$ were Grade 3 (G3) with $15.8 \%$ attributable to T-VEC and $21.1 \%$ attributable to ipilimumab; no dose-limiting toxicities (DLT) were observed. Subsequently, a phase II study was performed in which 198 patients with unresectable advanced melanoma were randomized to T-VEC plus ipilmumab or ipilumab monotherapy. (35) The study met its primary endpoint with a significantly higher ORR observed with combination therapy when compared to ipilimumab alone (39\% versus $18 \%$; odds ratio, 2.9; $\mathrm{p}<0.002$ ). The most common AEs were fatigue, chills, and diarrhea with $45 \%$ G3 AEs seen in combination therapy versus $35 \%$ with ipilimumab alone.

In a phase Ib study (MASTERKEY-265), 21 patients with unresectable stage IIIB-IVM1c melanoma were enrolled to receive combination treatment with T-VEC plus pembrolizumab. The most common AEs were fatigue, pyrexia, and chills.(36) Median time to response was 17 weeks, the ORR was $48 \%$ and the CR was $14 \%$ with confirmation by per modified 
immune-related response criteria (irRC) (unconfirmed ORR $=57 \%$; $\mathrm{CR}=24 \%$ ). A phase III randomized controlled trial (KEYNOTE-034) evaluating T-VEC (versus intralesional placebo) plus pembrolizumab is currently ongoing (NCT02263508).(37) T-VEC is also being investigated for use as neoadjuvant therapy. In a phase II study, 150 patients with completely resectable stage IIIB- IVM1 a melanoma were randomized to treatment with adjuvant T-VEC prior to surgical resection versus surgery alone. This trial has recently concluded and the results are pending.(38)

Currently T-VEC is injected into visible, palpable, or identifiable lesions using bedside ultrasound guidance. There are ongoing trials to develop alternative delivery strategies to visceral sites.(39) Early safety data from a phase I open-label trial of T-VEC injected into liver tumors using ultrasound under CT guidance showed tolerability and feasibility. $(40,41)$ Liver tumors were either primary or metastatic, including some that were metastatic melanoma. There were $28.6 \% \mathrm{G} 3$ treatment related AEs. A DLT was reported after 1 dose for a G3 increase in AST and a G2 increase in bilirubin. Treatment-related serious AEs included AST increase $(n=1)$, ALT increase $(n=1)$, and nausea $(n=1)$. Procedure-related adverse events included: liver cholestasis due to hematoma $(\mathrm{n}=1)$ and hepatic hemorrhage $(\mathrm{n}=1$.) A phase Ib/II trial (MASTERKEY-318) set to accrue 244 patients is underway, in which T-VEC, alone or in combination with systemic IV pembrolizumab, is directly injected under CT/US guidance into different liver tumor types, including hepatic metastasis from melanoma (NCT02509507.)(39, 42)

HF-10-HF10 is an attenuated, replication-competent, and spontaneously occurring HSV type 1 mutant OV with high tumor selectivity. $(43,44)$ UL53 is a gamma accessory gene which is overexpressed by HF10 and encodes glycoprotein $\mathrm{gK}$ which regulates HSV egression from infected cells.(45) UL56 is a gamma accessory gene which is involved in the pathogenicity and latency of HSV-1 and loss of UL56 in HF10 significantly decreases neuroinvasiveness of the virus.(45) It also lacks latency associated transcripts (LATs), which play a role in the reactivation of the HSV-1 virus.(45)

HF10 has shown potential in the preclinical setting against breast, head and neck squamous cell carcinoma, pancreatic cancer, and melanoma.(30, 45-47) In a murine melanoma model using DBA/2 mice and clone M3 cells, intratumoral administration of HF-10 resulted in a reduction in tumor mass, while intraperitoneal injection was shown to produce a $100 \%$ survival rate.(48) In a murine B16 melanoma model, increased survival and reduced tumor growth were observed.(49)

In a phase I clinical trial examining the effects of HF10 on superficial and refractory cancers in 24 patients, including malignant melanoma, chills, nausea, fatigue and weight loss were observed in $12.5 \%$ of patients with $87.5 \%$ of all patients experiencing tumor emergent adverse events.(50) A phase II clinical trial of 46 patients ranging 28 to 91 years of age, using HF10 in combination with ipilimumab in stage IIIB/IV unresectable melanoma had a reported median best ORR of $41 \%$ at 24 weeks, PFS of 19 months, and median overall survival of 21.8 months.(47) Three patients experienced $\geq$ Grade 3 (G3) adverse events as a result of HF10. $37 \%$ of patients experienced $\geq \mathrm{G} 3$ adverse events as a result of ipilimumab. 
A phase II clinical trial combining neoadjuvant HF-10 and nivolumab is currently recruiting patients.(51)

\section{Oncolytic Coxsackieviruses}

Coxsackieviruses have also been shown to possess promising potential as effective oncolytic therapies for melanoma. They first garnered interest as a possible cancer treatment based on to their known interactions with intercellular adhesion molecule-1 (ICAM-1) and decayaccelerating factor (DAF) receptors, both of which have been shown to be markedly overexpressed in melanoma.(52) The effects of coxsackieviruses on human melanoma cells were first described by Shafren et al. (2004), who showed that the genetically unmodified wild-type coxsackievirus A21 (CVA21) induced oncolysis of both in vitro cell lines and human melanoma xenografts.(53) It was later found that several other group A coxsackieviruses, specifically CVA13, 15, and 18, also demonstrated oncolytic activity in melanoma cells and tumors. Furthermore, sera from patients with stage IV melanoma had undetectable levels of protective neutralizing antibodies for serotypes 13,15 , and 18 , while several patients had low levels of antibodies against CVA21, suggesting that a combinatorial approach may be helpful for patients with prior exposure to these viruses.(54)

CVA21, commercially known as CAVATAK, is currently manufactured as an intratumoral agent and has completed phase II clinical trial studies in patients with stage IIIC and IV melanoma. Of the 57 patients enrolled, 22 patients (38.6\%) displayed immune-related progression free survival at 6 month with no serious adverse events attributable to CVA21. (55) A phase II study assessing extended dosing finished recruitment in 2016 and data analysis is currently underway.(56) Combination therapy with intratumoral CVA21 and immune checkpoint inhibitors, specifically ipilimumab and pembrolizumab, are also being studied in patients with advanced melanoma, with preliminary results reporting ORRs around $50 \%$ with no DLTs. $(57,58)$

\section{Other Oncolytic Viruses}

While HSV and coxsackievirus have gained much attention, several other oncolytic viruses have also shown early promising results in melanoma. Reovirus is a double-stranded RNA virus of the Reoviridae family whose anti-tumor properties stem from its ability to selectively infect and kill cells with overactive Ras signaling.(59) Reolysin, a serotype-3dearing reovirus strain, has been evaluated in the treatment of metastatic melanoma. Reolysin was shown to be safe and well-tolerated in three phase I trials of patients with advanced solid tumors.(59-61) However, a phase II study of Reolysin in 21 patients with metastatic melanoma showed no objective responses when given intravenously.(62) Severe (grade 3-4) toxicities were rare but included fatigue, lymphocytopenia, and hyponatremia, while the same toxicities were commonly observed but with low grade. In another phase II study, Reolysin was evaluated in combination with carboplatin and paclitaxel in 14 patients with advanced melanoma.(63) The results from this study demonstrated an ORR of $21 \%$ and an OS of 5.2 and 10.9 months, respectively. This suggests improved clinical benefit over paclitaxel/carboplatin alone, which showed PFS and OS of 3 and 9 months, respectively, in other clinical trials. $(64,65)$ Toxicities of combination therapy were comparable to those of chemotherapy alone, although pyrexia was common and 1 case of G3 febrile neutropenia 
was observed. There is currently insufficient evidence to recommend use of reovirus in the treatment of metastatic melanoma, however, combination therapy with Reolysin remains a potential therapeutic option and should be evaluated in the clinical setting.

Rigvir, an unmodified, single-stranded RNA ECHO-7 virus of the Picornaviridae family, is approved in Latvia and Georgia for oncolytic virotherapy. However, there is currently limited evidence of its safety and efficacy. Rigvir targets CD55/DAF-3, a GPI-anchored protein present on cancer cells, and has the ability to elicit both humoral and T-cell-mediated anti-tumor responses. $(66,67)$ A retrospective study of Rigvir in patients with stage IB-IIC melanoma found that Rigvir significantly prolonged survival and reduced mortality, without record of any "untoward" side effects(68), and a case report showed prolonged survival in patients with stage IV M1c melanoma treated with Rigvir.(69) The case report data has limited applicability, as it was obtained retrospectively and lacked a control arm. However, given the potential specificity of this therapy for cancer cells, the safety and efficacy of Rigvir in the treatment of melanoma merits further investigation.

Several trials of recombinant vaccinia virus have been conducted in advanced melanoma, with promising early results. Vaccinia virus is a double-stranded DNA virus of the poxvirus family, and can be modified to express both tumor-specific antigens and costimulatory molecules. A phase I trial in which vaccinia virus expressing the CD80 costimulatory molecule was injected into lesions, achieved an objective clinical response of $25 \%$ in 12 patients with metastatic melanoma with only low-grade toxicities, and found an enhanced $\mathrm{T}$ cell systemic response against the melanoma-specific antigens gp100 and MART-1.(70) A similar trial was conducted with intralesional injections of vaccinia virus simultaneously expressing 3 costimulatory molecules (B7.1, ICAM-1, LFA03), and found an objective clinical response of 53\%, also with limited low-grade toxicities.(71) Two phase I/II trials in patients with advanced melanoma have assessed the safety and efficacy of vaccinia virus expressing both melanoma tumor-specific antigens (MART-1, pg100, tyrosinase $1-9$ ) and costimulatory molecules (CD80, CD86) given intradermally and intranodally. $(72,73)$

Both studies observed an increase in tumor-specific cytotoxic T lymphocyte (CTL) responses, and objective clinical responses of $41 \%$ (intradermal) and 50\% (intranodal). Side effects were low-grade, with 1 episode of G3 leukopenia. In sum, vaccinia virus appears to be safe and effective in the treatment of advanced melanoma, and these results are encouraging with respect to both the clinical response as well as the specificity of the immune response.

Prime-boost is a technique that employs two different OVs to reduce viral clearance by neutralizing antibodies and to "boost" the immune response to tumor antigen. $(74,75) \mathrm{A}$ recent study found that prime-boost in a murine model with B16 melanoma using systemic Reovirus/Vesicular Stomatitis Virus prime-boost treatment in combination with anti-PD-1 therapy showed significantly improved survival with long-term cures.(76) This could be another avenue to explore in treatment of human melanoma.

Adenovirus is a double-stranded DNA virus of the Adenoviridae family, which has been studied to a limited degree in the treatment of human melanoma. A phase I study of 14 
patients with unresectable stage III/IV melanoma injected intratumorally with adenovirus expressing the cytokine IL-12 induced biologic and clinical responses, and was welltolerated (77). A clinical study of nine patients with chemotherapy refractory melanoma evaluated the efficacy of adenovirus expressing GM-CSF. Four of nine patients were evaluable for clinical response; one patient demonstrated tumor shrinkage, and two had stable disease. Side effects were mostly low-grade, including constitutional symptoms, nausea, and pain, with three instances of high-grade fatigue, dyspnea, and pericardial/pleura fluid, all caused by disease progression (78). Data on the efficacy of adenovirus in human melanoma is limited and merits further study.

\section{Current Challenges}

\section{OV Efficacy and Tumor Resistance}

Several factors can limit OV efficacy and promote tumor escape. Though OVs are intended to generate a tumor-specific local and systemic immune response, delivery can be unpredictable and have unintended consequences. Initial inoculum size and time to peak viral levels may vary.(4) The inoculum may face significant barriers to intratumoral dissemination such as heterogeneous tumor perfusion and high interstitial pressures. $(3,79)$

The ability of the host to mount an efficient antiviral defense is a potential mechanism of resistance that may restrict therapeutic efficacy. Pre-existing antibodies may also neutralize the virus before tumor cell invasion.(3) Though tumor cell death may incite a strong immune response, this immune response may clear the virus, limiting further anti-tumor activity.(3) Furthermore, systemic immune responses arising from local tumor antigens may not be effective against often heterogeneous metastatic lesions.(80)

Interferons play a crucial role in the viral defense. Moreover, OVs can trigger an innate immune response resulting in rapid clearance of the virus.(81) This has led to the idea that initial inhibition of immune responses could facilitate viral proliferation.(82) Besides the immune system, other proteins such as vascular endothelial growth factor, whose presence was shown to limit efficacy of an OV derived from HSV,(83) seem to play a role in the viral replication.

Physical barriers also lower the efficacy of OVs. Such barriers can be tumor cellular structure and/or the extracellular matrix (ECM). The ECM is a complex dense network consisting of proteins, glycoproteins, and polysaccharides.(84) Several viruses use cellular surface receptors to enter cells. Tumors of epithelial origin often retain the firm intercellular configuration seen in the tissue they originate from. These tight junctions can conceal cellular receptors from the virus.(85)

\section{Clinical Translation and Safety}

Clinicians will require additional education and training in OV handling, preparation, and delivery as these therapies continue their expansion into the clinic.(86) Concerns pertaining to biosafety are relevant to all OVs and special precautions must be taken in their administration, especially for live OVs such as T-VEC.(39) Pregnant women and 
immunocompromised individuals should not administer the virus, and practitioners should use personal protective equipment when injecting T-VEC.(39)

In most cases, OVs are directly injected into a readily accessible tumor. T-VEC is typically administered in the outpatient setting into lesions that can be visualized, palpated, or easily reached using bedside ultrasound.(86) Practitioners must also learn specific injection techniques and maneuvers used to achieve optimal virus delivery and tumor perfusion. Delivery to less accessible tumors using an image-guided approach is being investigated, as well as optimization of intravenous (IV) delivery, which frequently results in hepatic sequestration or limited OV efficacy.

Our experience with T-VEC has demonstrated that it can be challenging for physicians to decide whether or not to continue treatment as initial response may be delayed and distinguishing pseudoprogression from true progression is often difficult. Furthermore, injected metastatic lesions may remain black, making it difficult to determine if the discoloration is due to residual melanoma or melanophages (macrophages that have phagocytozed pigmented compounds), even after biopsy and tissue staining with melanoma specific markers.(87) Techniques such as multiplex immunofluorescence can help distinguish melanophages from melanoma and are being investigated.(87)

T-VEC is typically administered with an initial dose of up to $4 \mathrm{ml} 10^{6} \mathrm{PFU} / \mathrm{ml}$ and is followed 3 weeks later by a higher viral load of up to $4 \mathrm{ml}$ of $10^{8} \mathrm{PFU} / \mathrm{ml}$. Treatment is given every 2 weeks until lesion resolution, lesion progression, or toxicity.(86) A lack of a complete response in the majority of treated patients with T-VEC monotherapy has prompted some to consider the possibility that changes in dose and/or dosing schedule may improve OV efficacy.(80)

\section{Conclusions}

Although the potential for viruses to induce an antitumor immune response has been acknowledged for years, only recently has oncolytic virotherapy become a practical reality in the treatment of cancer. T-VEC has been a breakthrough in the treatment of melanoma, but also brings its own challenges with which clinicians must contend as it transitions to the clinic. Combination therapy is an active area of research and several pre-clinical studies are underway. Strategies that pair highly immunogenic OVs with immune-checkpoint inhibitors are especially promising. Thus far, treatment with T-VEC and related agents appears to avoid serious life-threatening toxicities. Safety data for combination therapy is also emerging. As OV therapy continues to gain momentum, and more patients are treated with OVs at various doses and in combination with different agents, the full scope of OV potential and related toxicities will be better understood.

\section{References}

1. Fountzilas C, Patel S, Mahalingam D. Review: Oncolytic virotherapy, updates and future directions. Oncotarget. 2017;8(60):102617-39. [PubMed: 29254276]

2. Martuza RL, Malick A, Markert JM, Ruffner KL, Coen DM. Experimental therapy of human glioma by means of a genetically engineered virus mutant. Science. 1991;252(5007):854-6. [PubMed: 1851332] 
3. Kaufman HL, Kohlhapp FJ, Zloza A. Oncolytic viruses: a new class of immunotherapy drugs. Nat Rev Drug Discov. 2015;14(9):642-62. [PubMed: 26323545]

4. Maroun J, Munoz-Alia M, Ammayappan A, Schulze A, Peng KW, Russell S. Designing and building oncolytic viruses. Future Virol. 2017;12(4):193-213. [PubMed: 29387140]

5. Yu Z, Chan MK, Oc P, Eisenberg DP, Shah JP, Singh B, et al. Enhanced nectin-1 expression and herpes oncolytic sensitivity in highly migratory and invasive carcinoma. Clin Cancer Res. 2005;11(13):4889-97. [PubMed: 16000587]

6. Hammond AL, Plemper RK, Zhang J, Schneider U, Russell SJ, Cattaneo R. Single-chain antibody displayed on a recombinant measles virus confers entry through the tumor-associated carcinoembryonic antigen. J Virol. 2001;75(5):2087-96. [PubMed: 11160713]

7. Rabinovich GA, Gabrilovich D, Sotomayor EM. Immunosuppressive strategies that are mediated by tumor cells. Annu Rev Immunol. 2007;25:267-96. [PubMed: 17134371]

8. Schaaf MB, Garg AD, Agostinis P. Defining the role of the tumor vasculature in antitumor immunity and immunotherapy. Cell Death Dis. 2018;9(2):115. [PubMed: 29371595]

9. Mansour M, Palese P, Zamarin D. Oncolytic specificity of Newcastle disease virus is mediated by selectivity for apoptosis-resistant cells. J Virol. 2011;85(12):6015-23. [PubMed: 21471241]

10. Small EJ, Carducci MA, Burke JM, Rodriguez R, Fong L, van Ummersen L, et al. A phase I trial of intravenous CG7870, a replication-selective, prostate-specific antigen-targeted oncolytic adenovirus, for the treatment of hormone-refractory, metastatic prostate cancer. Mol Ther. 2006;14(1):107-17. [PubMed: 16690359]

11. Guo ZS, Liu Z, Bartlett DL. Oncolytic Immunotherapy: Dying the Right Way is a Key to Eliciting Potent Antitumor Immunity. Front Oncol. 2014;4:74. [PubMed: 24782985]

12. Burrows FJ, Gore M, Smiley WR, Kanemitsu MY, Jolly DJ, Read SB, et al. Purified herpes simplex virus thymidine kinase retroviral particles: III. Characterization of bystander killing mechanisms in transfected tumor cells. Cancer Gene Ther. 2002;9(1):87-95. [PubMed: 11916247]

13. Rubartelli A, Lotze MT. Inside, outside, upside down: damage-associated molecular-pattern molecules (DAMPs) and redox. Trends Immunol. 2007;28(10):429-36. [PubMed: 17845865]

14. Kaufman HL, Amatruda T, Reid T, Gonzalez R, Glaspy J, Whitman E, et al. Systemic versus local responses in melanoma patients treated with talimogene laherparepvec from a multi-institutional phase II study. J Immunother Cancer. 2016;4:12. [PubMed: 26981242]

15. Prestwich RJ, Errington F, Diaz RM, Pandha HS, Harrington KJ, Melcher AA, et al. The case of oncolytic viruses versus the immune system: waiting on the judgment of Solomon. Hum Gene Ther. 2009;20(10):1119-32. [PubMed: 19630549]

16. de Vries CR, Kaufman HL, Lattime EC. Oncolytic viruses: focusing on the tumor microenvironment. Cancer Gene Ther. 2015;22(4):169-71. [PubMed: 25721204]

17. Guedan S, Rojas JJ, Gros A, Mercade E, Cascallo M, Alemany R. Hyaluronidase expression by an oncolytic adenovirus enhances its intratumoral spread and suppresses tumor growth. Mol Ther. 2010;18(7):1275-83. [PubMed: 20442708]

18. Li H, Haviv YS, Derdeyn CA, Lam J, Coolidge C, Hunter E, et al. Human immunodeficiency virus type 1-mediated syncytium formation is compatible with adenovirus replication and facilitates efficient dispersion of viral gene products and de novo-synthesized virus particles. Hum Gene Ther. 2001;12(18):2155-65. [PubMed: 11779400]

19. Toda M, Martuza RL, Rabkin SD. Tumor growth inhibition by intratumoral inoculation of defective herpes simplex virus vectors expressing granulocyte-macrophage colony-stimulating factor. Mol Ther. 2000;2(4):324-9. [PubMed: 11020347]

20. Mohit E, Rafati S. Chemokine-based immunotherapy: delivery systems and combination therapies. Immunotherapy. 2012;4(8):807-40. [PubMed: 22947009]

21. Bossow S, Grossardt C, Temme A, Leber MF, Sawall S, Rieber EP, et al. Armed and targeted measles virus for chemovirotherapy of pancreatic cancer. Cancer Gene Ther. 2011;18(8):598-608. [PubMed: 21701532]

22. Dharmadhikari N, Mehnert JM, Kaufman HL. Oncolytic virus immunotherapy for melanoma. Curr Treat Options Oncol. 2015;16(3):326. [PubMed: 25777572]

23. Ottolino-Perry K, Diallo JS, Lichty BD, Bell JC, McCart JA. Intelligent design: combination therapy with oncolytic viruses. Mol Ther. 2010;18(2):251-63. [PubMed: 20029399] 
24. Liu BL, Robinson M, Han ZQ, Branston RH, English C, Reay P, et al. ICP34.5 deleted herpes simplex virus with enhanced oncolytic, immune stimulating, and anti-tumour properties. Gene Ther. 2003;10(4):292-303. [PubMed: 12595888]

25. Brown SM, MacLean AR, Aitken JD, Harland J. ICP34.5 influences herpes simplex virus type 1 maturation and egress from infected cells in vitro. J Gen Virol. 1994;75 ( Pt 12):3679-86. [PubMed: 7996163]

26. He B, Chou J, Liebermann DA, Hoffman B, Roizman B. The carboxyl terminus of the murine MyD116 gene substitutes for the corresponding domain of the gamma(1)34.5 gene of herpes simplex virus to preclude the premature shutoff of total protein synthesis in infected human cells. J Virol. 1996;70(1):84-90. [PubMed: 8523596]

27. Fruh K, Ahn K, Djaballah H, Sempe P, van Endert PM, Tampe R, et al. A viral inhibitor of peptide transporters for antigen presentation. Nature. 1995;375(6530):415-8. [PubMed: 7760936]

28. York IA, Roop C, Andrews DW, Riddell SR, Graham FL, Johnson DC. A cytosolic herpes simplex virus protein inhibits antigen presentation to CD8+ T lymphocytes. Cell. 1994;77(4):525-35. [PubMed: 8187174]

29. van de Laar L, Coffer PJ, Woltman AM. Regulation of dendritic cell development by GM-CSF: molecular control and implications for immune homeostasis and therapy. Blood. 2012;119(15): 3383-93. [PubMed: 22323450]

30. Hu JC, Coffin RS, Davis CJ, Graham NJ, Groves N, Guest PJ, et al. A phase I study of OncoVEXGM-CSF, a second-generation oncolytic herpes simplex virus expressing granulocyte macrophage colony-stimulating factor. Clin Cancer Res. 2006;12(22):6737-47. [PubMed: 17121894]

31. Senzer NN, Kaufman HL, Amatruda T, Nemunaitis M, Reid T, Daniels G, et al. Phase II clinical trial of a granulocyte-macrophage colony-stimulating factor-encoding, second-generation oncolytic herpesvirus in patients with unresectable metastatic melanoma. J Clin Oncol. 2009;27(34):5763-71. [PubMed: 19884534]

32. Kaufman HL, Kim DW, DeRaffele G, Mitcham J, Coffin RS, Kim-Schulze S. Local and distant immunity induced by intralesional vaccination with an oncolytic herpes virus encoding GM-CSF in patients with stage IIIc and IV melanoma. Ann Surg Oncol. 2010;17(3):718-30. [PubMed: 19915919]

33. Andtbacka RH, Kaufman HL, Collichio F, Amatruda T, Senzer N, Chesney J, et al. Talimogene Laherparepvec Improves Durable Response Rate in Patients With Advanced Melanoma. J Clin Oncol. 2015;33(25):2780-8. [PubMed: 26014293] * The OPTiM phase III clinical trial, comparing treatment with T-VEC to GM-CSF, ultimately led to the FDA approval of the use of TVEC for the treatment of advanced melanoma. 436 patients with unresected stage IIIB to IV melanoma were randomized. DRR was $16.3 \%$ for patients treated with T-VEC, as opposed to $2.1 \%$ for patients treated with GM-CSF.

34. Puzanov I, Milhem MM, Minor D, Hamid O, Li A, Chen L, et al. Talimogene Laherparepvec in Combination With Ipilimumab in Previously Untreated, Unresectable Stage IIIB-IV Melanoma. J Clin Oncol. 2016;34(22):2619-26. [PubMed: 27298410] * This phase Ib trial reported that combining T-VEC and ipilimumab was safe and effective in patients with stage IIIB-IVM1c melanoma. There were G3/4 AEs in $26.3 \%$ of patients and no DLTs. ORR was $50 \%$, OS was $67 \%$, and 18-month PFS was 50\%.

35. Chesney J, Puzanov I, Collichio F, Singh P, Milhem MM, Glaspy J, et al. Randomized, Open-Label Phase II Study Evaluating the Efficacy and Safety of Talimogene Laherparepvec in Combination With Ipilimumab Versus Ipilimumab Alone in Patients With Advanced, Unresectable Melanoma. J Clin Oncol. 2017:JCO2017737379.** This is a phase II randomized trial of combined T-VEC and ipilimumab in unresectable stage IIIB-IV melanoma. The study met its primary endpoint. The ORR was significantly higher in patients receiving combination therapy (39\%), relative to patients receiving ipilimumab treatment alone $(18 \%)$.

36. Georgina V Long RD, Ribas Antoni, Puzanov Igor, VanderWalde Ari, Robert Hans Ingemar Andtbacka, Olivier Michielin, Anthony J. Olszanski, Josep Malvehy, Cebon Jonathan S., Eugenio Fernandez, Kirkwood John M., Gajewski Thomas, Gause Christine K., Chen Lisa, Gorski Kevin, Anderson Abraham, Kaufman David Ross, Chou Jeffrey, and Hodi F. Stephen. Efficacy analysis of MASTERKEY-265 phase $1 \mathrm{~b}$ study of talimogene laherparepvec (T-VEC) and pembrolizumab 
(pembro) for unresectable stage IIIB-IV melanoma. 2016;34(15):9568.* This abstract reported the results from the phase Ib/III clinical trial evaluating the efficacy, safety, and tolerability of combining T-VEC and pembrolizumab in patients with unresectable stage IIIB-IV melanoma. There is an ongoing phase III clinical trial.

37. Pembrolizumab With or Without Talimogene Laherparepvec or Talimogene Laherparepvec Placebo in Unresected Melanoma (KEYNOTE-034) [Internet]. 2014 Available from: https:// clinicaltrials.gov/ct2/show/NCT02263508.** This is an ongoing double blind randomized phase III clinical trial comparing the efficacy of T-VEC + pembrolizumab to pembrolizumab + placebo in stage IIIB-IVM1c melanoma. Awaiting results.

38. Robert Hans Ingemar Andtbacka MC, Li Ai, Shilkrut Mark, and Ross Merrick I. Phase 2, multicenter, randomized, open-label trial assessing efficacy and safety of talimogene laherparepvec (T-VEC) neoadjuvant treatment (tx) plus surgery vs surgery for resectable stage IIIB/C and IVM1a melanoma (MEL). Journal of Clinical Oncology 2015;33(15):TPS9094-TPS

39. Orloff M Spotlight on talimogene laherparepvec for the treatment of melanoma lesions in the skin and lymph nodes. Oncolytic Virother. 2016;5:91-8. [PubMed: 27785448]

40. Randolph Hecht MP J, Cubillo Antonio, Calvo Aitana, Raman Steven, Liu Chunxu, Chan Emily, Chesney Jason Alan, and Prat Aleix. Early safety from a phase 1, multicenter, open-label clinical trial of talimogene laherparepvec (T-VEC) injected into liver tumors. Journal of Clinical Oncology. 2018;36(4):438- [PubMed: 29240540]

41. J Randolph Hecht SR, Sze Daniel Y,Lockhart A Craig, Moss Rebecca A, Liu Kate, Chou Jeffrey and Reid Tony. A Phase I, multicenter, open-label trial to evaluate the safety of talimogene laherparepvec (T-VEC) injected into liver tumors.. J ImmunoTher Cancer. 2015;3:P180.

42. Trial to Evaluate the Safety of Talimogene Laherparepvec Injected Into Liver Tumors Alone and in Combination With Systemic Pembrolizumab (MASTERKEY-318) [Internet]. 2015 Available from: https://clinicaltrials.gov/ct2/show/NCT02509507.* There is a need for new methods of delivery of oncolytic viruses, especially to visceral sites of disease. This ongoing phase Ib study examines the safety of intrahepatic injection into liver tumors of T-VEC alone (under CT/US guidance), and of intrahepatic injection of T-VEC (under CT/US guidance) in combination with pembrolizumab.

43. Ushijima Y, Luo C, Goshima F, Yamauchi Y, Kimura H, Nishiyama Y. Determination and analysis of the DNA sequence of highly attenuated herpes simplex virus type 1 mutant HF10, a potential oncolytic virus. Microbes Infect. 2007;9(2):142-9. [PubMed: 17218138]

44. Nishiyama Y, Kimura H, Daikoku T. Complementary lethal invasion of the central nervous system by nonneuroinvasive herpes simplex virus types 1 and 2. J Virol. 1991;65(8):4520-4. [PubMed: 1649347]

45. Sahin TT, Kasuya H, Nomura N, Shikano T, Yamamura K, Gewen T, et al. Impact of novel oncolytic virus HF10 on cellular components of the tumor microenviroment in patients with recurrent breast cancer. Cancer Gene Ther. 2012;19(4):229-37. [PubMed: 22193629]

46. Nakao A, Kasuya H, Sahin TT, Nomura N, Kanzaki A, Misawa M, et al. A phase I dose-escalation clinical trial of intraoperative direct intratumoral injection of HF10 oncolytic virus in nonresectable patients with advanced pancreatic cancer. Cancer Gene Ther. 2011;18(3):167-75. [PubMed: 21102422]

47. Robert Hans Ingemar Andtbacka MIR, Agarwala Sanjiv S., Taylor Matthew H., Vetto John T., Neves Rogerio Izar, Daud Adil, Khong Hung T., Ungerleider Richard S., Tanaka Maki, and Grossmann Kenneth F. Final results of a phase II multicenter trial of HF10, a replicationcompetent HSV-1 oncolytic virus, and ipilimumab combination treatment in patients with stage IIIB-IV unresectable or metastatic melanoma.. Journal of Clinical Oncology. 2017;35(15).** This phase II clinical trial evaluated the efficacy of combining ipilimumab with HF10 (an oncolytic virus) in stage IIIB-IV unresectable or metastatic melanoma. Reported median best ORR was $41 \%$ at 24 weeks, PFS was 19 months, and the median overall survival was 21.8 months.

48. Watanabe D, Goshima F, Mori I, Tamada Y, Matsumoto Y, Nishiyama Y. Oncolytic virotherapy for malignant melanoma with herpes simplex virus type 1 mutant HF10. J Dermatol Sci. 2008;50(3): 185-96. [PubMed: 18226503]

49. Takakuwa H, Goshima F, Nozawa N, Yoshikawa T, Kimata H, Nakao A, et al. Oncolytic viral therapy using a spontaneously generated herpes simplex virus type 1 variant for disseminated 
peritoneal tumor in immunocompetent mice. Arch Virol. 2003;148(4):813-25. [PubMed: 12664303]

50. RLea Ferris. Phase I trial of intratumoral therapy using HF10, an oncolytic HSV-1, demonstrates safety in HSV+/HSV- patients with refractory and superficial cancers.. J Clin Oncol 2014;32.

51. Neoadjuvant Trial of Nivolumab in Combination With HF10 Oncolytic Viral Therapy in Resectable Stage IIIB, IIIC, IVM1a Melanoma [Internet]. 2017 Available from: https:// clinicaltrials.gov/ct2/show/NCT03259425?term=HF-10\&cond=melanoma\&rank=3.* Results from this ongoing phase II study evaluating the safety and efficacy of a treatment consisting of neoadjuvant HF-10 used in combination with nivolumab in patients with stage IIIB, IIIC, and IVM1a melanoma will be of importance.

52. Kageshita T, Yoshii A, Kimura T, Kuriya N, Ono T, Tsujisaki M, et al. Clinical relevance of ICAM-1 expression in primary lesions and serum of patients with malignant melanoma. Cancer Res. 1993;53(20):4927-32. [PubMed: 8104688]

53. Shafren DR, Au GG, Nguyen T, Newcombe NG, Haley ES, Beagley L, et al. Systemic therapy of malignant human melanoma tumors by a common cold-producing enterovirus, coxsackievirus a21. Clin Cancer Res. 2004;10(1 Pt 1):53-60. [PubMed: 14734451]

54. Au GG, Beagley LG, Haley ES, Barry RD, Shafren DR. Oncolysis of malignant human melanoma tumors by Coxsackieviruses A13, A15 and A18. Virol J. 2011;8:22. [PubMed: 21241513]

55. A Study of Intratumoral CAVATAK ${ }^{\mathrm{TM}}$ in Patients With Stage IIIc and Stage IV Malignant Melanoma (VLA-007 CALM ) (CALM) [Internet]. 2010 Available from: https:// clinicaltrials.gov/ct2/show/NCT01227551.

56. Efficacy and Safety of Intratumoral CAVATAK in Patients With Stage IIIc or IV Malignant Melanoma to Extend Dosing to 48 Weeks [Internet]. 2012 Available from: https:// clinicaltrials.gov/ct2/show/NCT01636882.

57. Curti B RJ, Hallmeyer S, et al. The MITCI (phase Ib) study: a novel immunotherapy combination of intralesional Coxsackievirus A21 and systemic ipilimumab in advanced melanoma patients with or without previous immune checkpoint therapy treatment. 2017 AACR Annual Meeting; April 25; Washington, DC 2017.* The preliminary results of this phase Ib clinical trial indicate that the novel combination of CVA21, a coxsackievirus, and ipilimumab is well-tolerated and leads to a durable response in patients with advanced melanoma. Preliminary ORR (50\%) was greater than the published data on these agents as monotherapy in the treatment of advanced melanoma.

58. Kaufman HL SK, Mehnert J, et al. Phase Ib study of intratumoral oncolytic coxsackievirus A21 (CVA21) and pembrolizumab in subjects with advanced melanoma.. 2016;27(suppl_6):1.* The results from this ongoing phase Ib clinical study combining CVA21 and pembrolizumab therapy in patients with advanced melanoma will be of importance.

59. Errington F, White CL, Twigger KR, Rose A, Scott K, Steele L, et al. Inflammatory tumour cell killing by oncolytic reovirus for the treatment of melanoma. Gene Ther. 2008;15(18):1257-70. [PubMed: 18401435]

60. Vidal L, Pandha HS, Yap TA, White CL, Twigger K, Vile RG, et al. A phase I study of intravenous oncolytic reovirus type 3 Dearing in patients with advanced cancer. Clin Cancer Res. 2008;14(21): 7127-37. [PubMed: 18981012]

61. Morris DG, Feng X, DiFrancesco LM, Fonseca K, Forsyth PA, Paterson AH, et al. REO-001: A phase I trial of percutaneous intralesional administration of reovirus type 3 dearing $(\operatorname{Reolysin}(\mathrm{R}))$ in patients with advanced solid tumors. Invest New Drugs. 2013;31(3):696-706. [PubMed: 22886613]

62. Galanis E, Markovic SN, Suman VJ, Nuovo GJ, Vile RG, Kottke TJ, et al. Phase II trial of intravenous administration of Reolysin((R)) (Reovirus Serotype-3-dearing Strain) in patients with metastatic melanoma. Mol Ther. 2012;20(10):1998-2003. [PubMed: 22871663]

63. Mahalingam D, Fountzilas C, Moseley J, Noronha N, Tran H, Chakrabarty R, et al. A phase II study of REOLYSIN $((\mathrm{R}))$ (pelareorep) in combination with carboplatin and paclitaxel for patients with advanced malignant melanoma. Cancer Chemother Pharmacol. 2017;79(4):697-703. [PubMed: 28289863]

64. Rao RD, Holtan SG, Ingle JN, Croghan GA, Kottschade LA, Creagan ET, et al. Combination of paclitaxel and carboplatin as second-line therapy for patients with metastatic melanoma. Cancer. 2006;106(2):375-82. [PubMed: 16342250] 
65. Hodi FS, Soiffer RJ, Clark J, Finkelstein DM, Haluska FG. Phase II study of paclitaxel and carboplatin for malignant melanoma. Am J Clin Oncol. 2002;25(3):283-6. [PubMed: 12040289]

66. Glinkina LS, Bruvere R, Venskus DR, Garklava RR, Muceniece AJ. [The cellular immunity indices of patients with malignant melanoma using the viral immunomodulator rigvir]. Vopr Onkol. 1992;38(5):540-7. [PubMed: 1300752]

67. Glinkina LS, Heisele OG, Garklava RR, Muceniece AJ. [The humoral immunity indices of patients with malignant skin melanoma using the viral immunomodulator rigvir]. Vopr Onkol. 1992;38(5): 534-40. [PubMed: 1300751]

68. Donina S, Strele I, Proboka G, Auzins J, Alberts P, Jonsson B, et al. Adapted ECHO-7 virus Rigvir immunotherapy (oncolytic virotherapy) prolongs survival in melanoma patients after surgical excision of the tumour in a retrospective study. Melanoma Res. 2015;25(5):421-6. [PubMed: 26193376]

69. Alberts P, Olmane E, Brokane L, Krastina Z, Romanovska M, Kupcs K, et al. Long-term treatment with the oncolytic ECHO-7 virus Rigvir of a melanoma stage IV M1c patient, a small cell lung cancer stage IIIA patient, and a histiocytic sarcoma stage IV patient-three case reports. APMIS. 2016;124(10):896-904. [PubMed: 27457663]

70. Kaufman HL, Deraffele G, Mitcham J, Moroziewicz D, Cohen SM, Hurst-Wicker KS, et al. Targeting the local tumor microenvironment with vaccinia virus expressing B7.1 for the treatment of melanoma. J Clin Invest. 2005;115(7):1903-12. [PubMed: 15937544]

71. Kaufman HL, Cohen S, Cheung K, DeRaffele G, Mitcham J, Moroziewicz D, et al. Local delivery of vaccinia virus expressing multiple costimulatory molecules for the treatment of established tumors. Hum Gene Ther. 2006;17(2):239-44. [PubMed: 16454657]

72. Adamina M, Rosenthal R, Weber WP, Frey DM, Viehl CT, Bolli M, et al. Intranodal immunization with a vaccinia virus encoding multiple antigenic epitopes and costimulatory molecules in metastatic melanoma. Mol Ther. 2010;18(3):651-9. [PubMed: 19935776]

73. Zajac P, Oertli D, Marti W, Adamina M, Bolli M, Guller U, et al. Phase I/II clinical trial of a nonreplicative vaccinia virus expressing multiple HLA-A0201-restricted tumor-associated epitopes and costimulatory molecules in metastatic melanoma patients. Hum Gene Ther. 2003;14(16): 1497-510. [PubMed: 14577912]

74. Zamarin D, Vigil A, Kelly K, Garcia-Sastre A, Fong Y. Genetically engineered Newcastle disease virus for malignant melanoma therapy. Gene Ther. 2009;16(6):796-804. [PubMed: 19242529]

75. Niu Z, Bai F, Sun T, Tian H, Yu D, Yin J, et al. Recombinant Newcastle Disease virus Expressing IL15 Demonstrates Promising Antitumor Efficiency in Melanoma Model. Technol Cancer Res Treat. 2015;14(5):607-15. [PubMed: 24645750]

76. Pecora AL, Rizvi N, Cohen GI, Meropol NJ, Sterman D, Marshall JL, et al. Phase I trial of intravenous administration of PV701, an oncolytic virus, in patients with advanced solid cancers. $\mathrm{J}$ Clin Oncol. 2002;20(9):2251-66. [PubMed: 11980996]

77. Gerald P Linette OH, Whitman Eric D., Nemunaitis John J., Chesney Jason, Agarwala Sanjiv S., Starodub Alexander, Barrett John A, Marsh Andrew, Martell Lori A., Cho Angela, Thomas D. Reed Hagop Youssoufian, Andrea Vergara-Silva. A phase I open-label study of Ad-RTS-hIL-12, an adenoviral vector engineered to express hIL-12 under the control of an oral activator ligand, in subjects with unresectable stage III/IV melanoma. J Clin Oncology. 2013;31.

78. Bramante S, Kaufmann JK, Veckman V, Liikanen I, Nettelbeck DM, Hemminki O, et al. Treatment of melanoma with a serotype 5/3 chimeric oncolytic adenovirus coding for GM-CSF: Results in vitro, in rodents and in humans. Int J Cancer. 2015;137(7):1775-83. [PubMed: 25821063]

79. Shen BH, Hermiston TW. Effect of hypoxia on Ad5 infection, transgene expression and replication. Gene Ther. 2005;12(11):902-10. [PubMed: 15690062]

80. Breitbach CJ, Lichty BD, Bell JC. Oncolytic Viruses: Therapeutics With an Identity Crisis. EBioMedicine. 2016;9:31-6. [PubMed: 27407036]

81. Fulci G, Dmitrieva N, Gianni D, Fontana EJ, Pan X, Lu Y, et al. Depletion of peripheral macrophages and brain microglia increases brain tumor titers of oncolytic viruses. Cancer Res. 2007;67(19):9398-406. [PubMed: 17909049] 
82. Fulci G, Breymann L, Gianni D, Kurozomi K, Rhee SS, Yu J, et al. Cyclophosphamide enhances glioma virotherapy by inhibiting innate immune responses. Proc Natl Acad Sci U S A. 2006;103(34):12873-8. [PubMed: 16908838]

83. Arulanandam R, Batenchuk C, Angarita FA, Ottolino-Perry K, Cousineau S, Mottashed A, et al. VEGF-Mediated Induction of PRD1-BF1/Blimp1 Expression Sensitizes Tumor Vasculature to Oncolytic Virus Infection. Cancer Cell. 2015;28(2):210-24. [PubMed: 26212250]

84. Lu P, Weaver VM, Werb Z. The extracellular matrix: a dynamic niche in cancer progression. J Cell Biol. 2012;196(4):395-406. [PubMed: 22351925]

85. Coyne CB, Bergelson JM. CAR: a virus receptor within the tight junction. Adv Drug Deliv Rev. 2005;57(6):869-82. [PubMed: 15820557]

86. Bommareddy PK, Silk AW, Kaufman HL. Intratumoral Approaches for the Treatment of Melanoma. Cancer J. 2017;23(1):40-7. [PubMed: 28114253]

87. Bayan C, Gartrell R, Lopez AT, Pradhan J, Chen C, Niedt G, et al. Distinguishing Melanophages from Melanoma in Metastatic Melanoma Treated with T-Vec: A Clinical Application Of Quantitative Multiplex Immunofluorescence. Journal of Investigative Dermatology. (in press). 


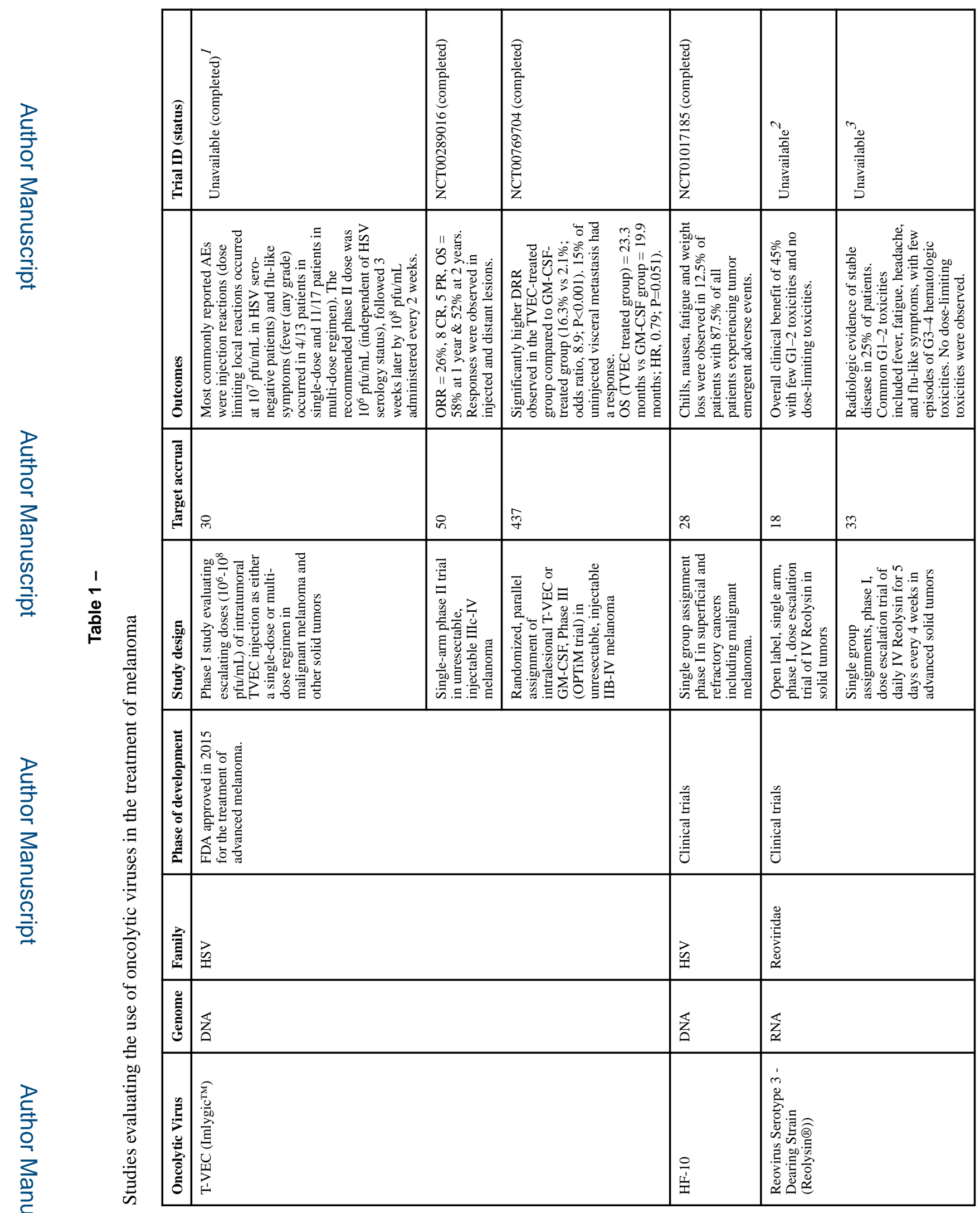

Curr Oncol Rep. Author manuscript; available in PMC 2019 August 25. 


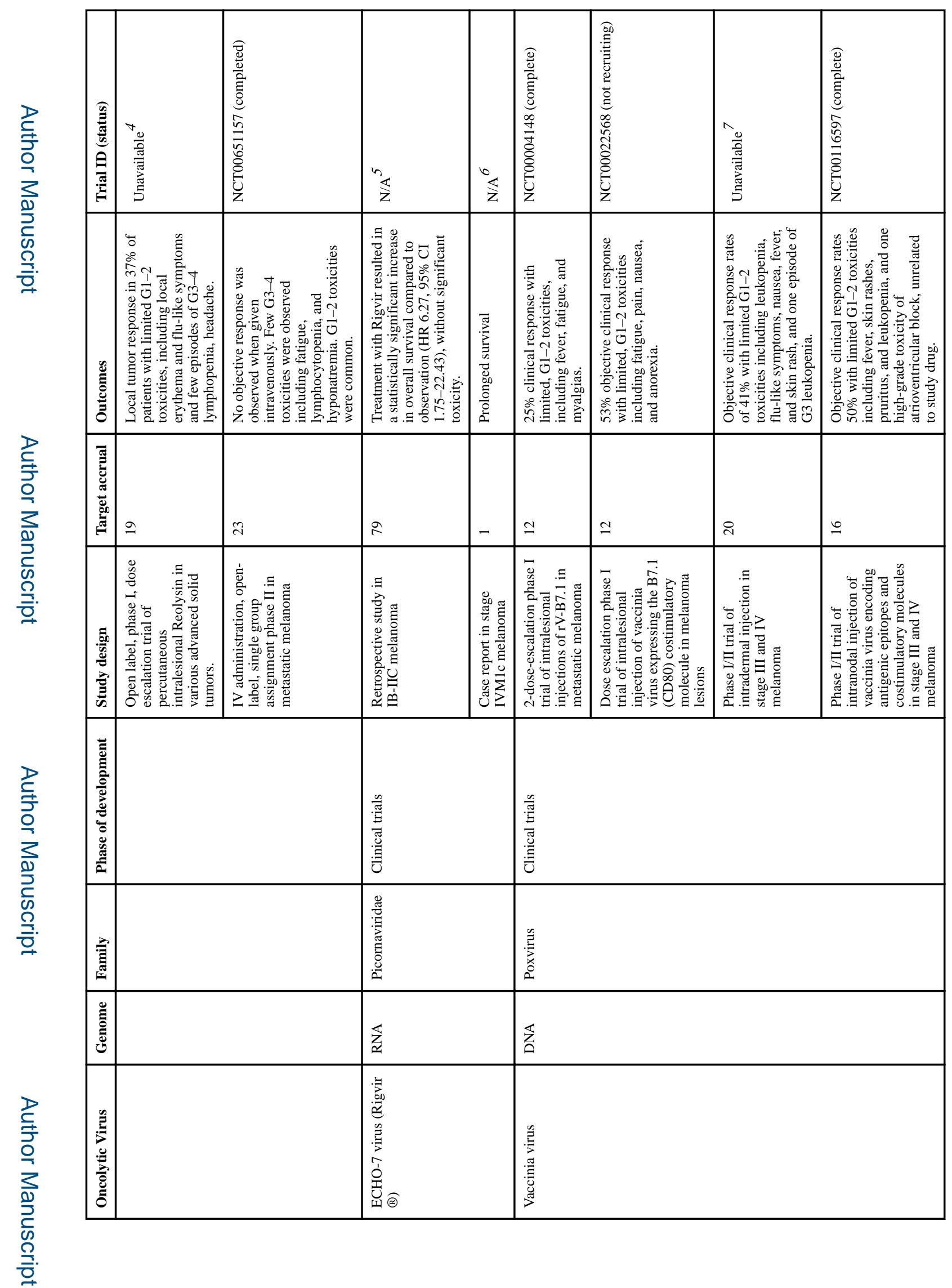

Curr Oncol Rep. Author manuscript; available in PMC 2019 August 25. 


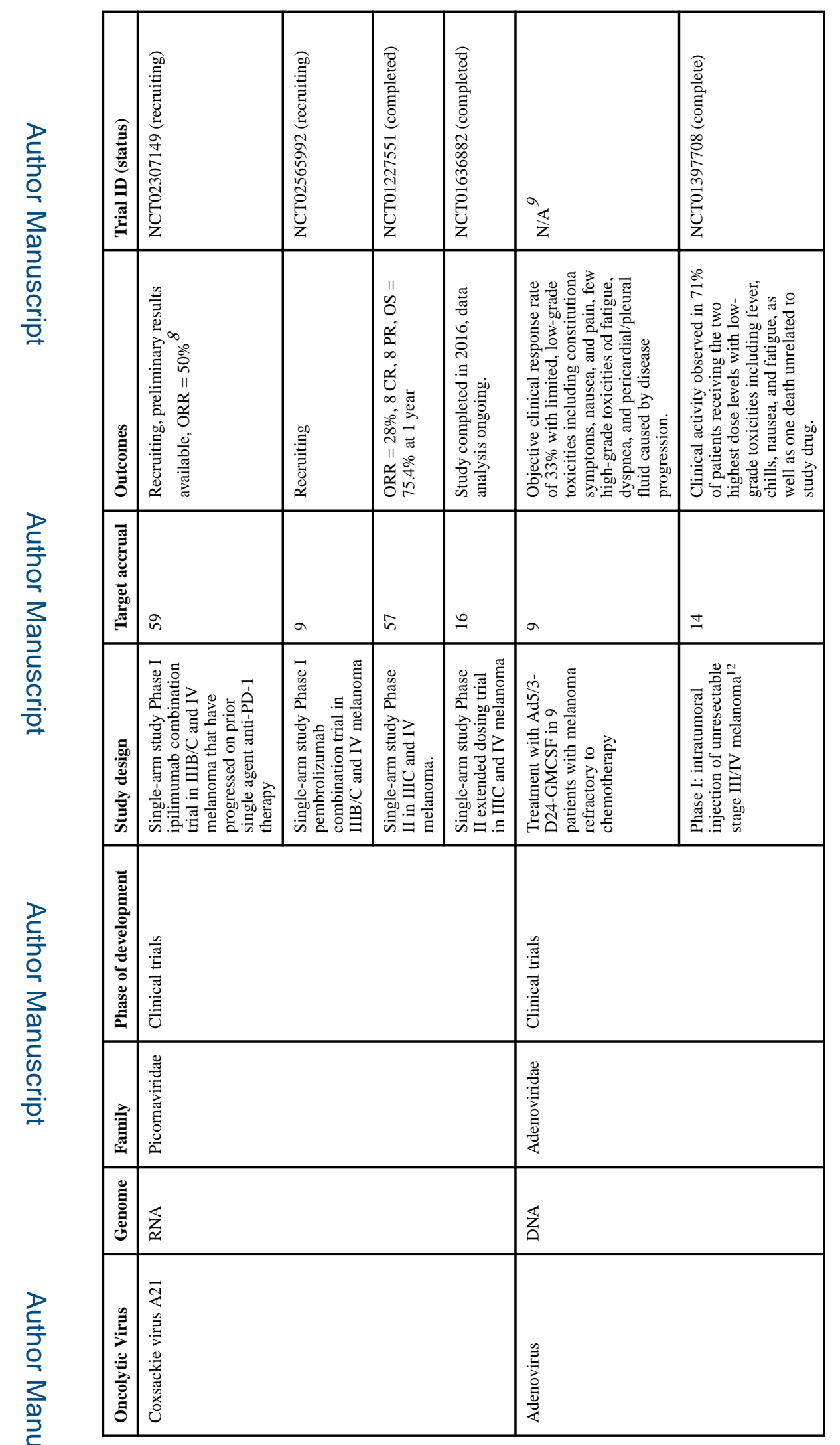

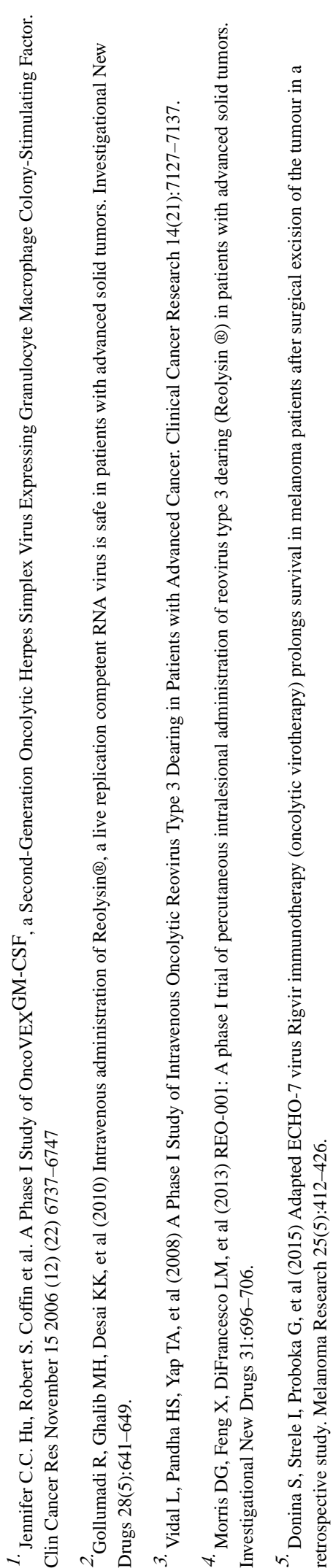

Curr Oncol Rep. Author manuscript; available in PMC 2019 August 25. 


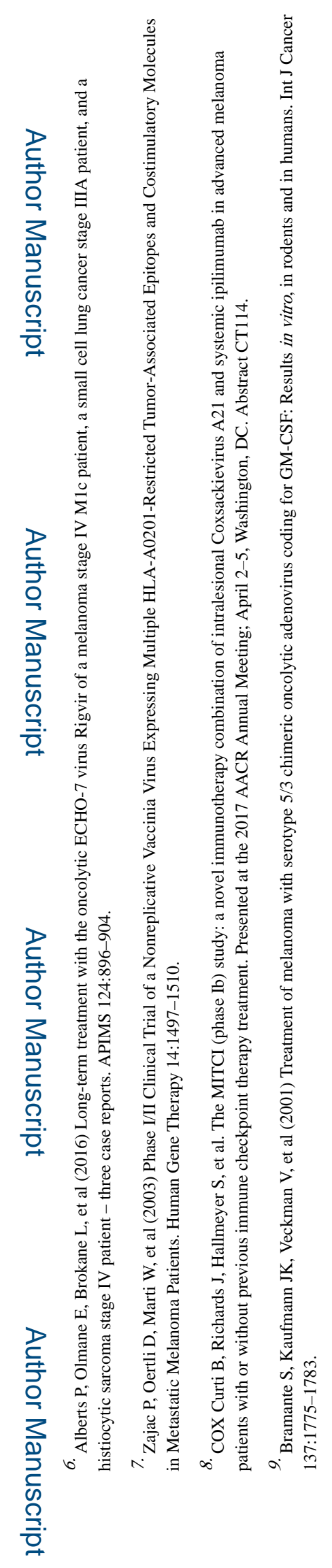

Curr Oncol Rep. Author manuscript; available in PMC 2019 August 25. 


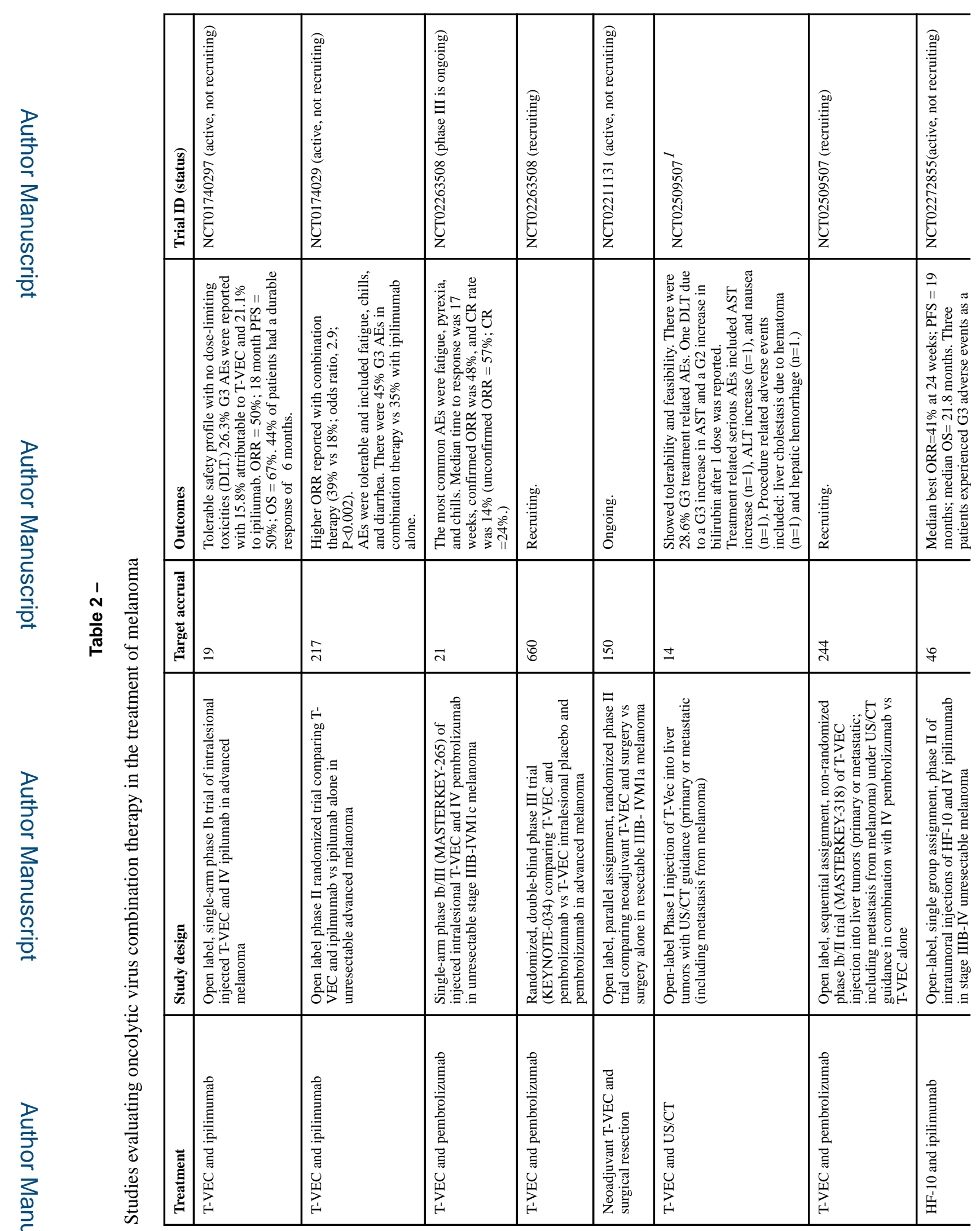

Curr Oncol Rep. Author manuscript; available in PMC 2019 August 25. 


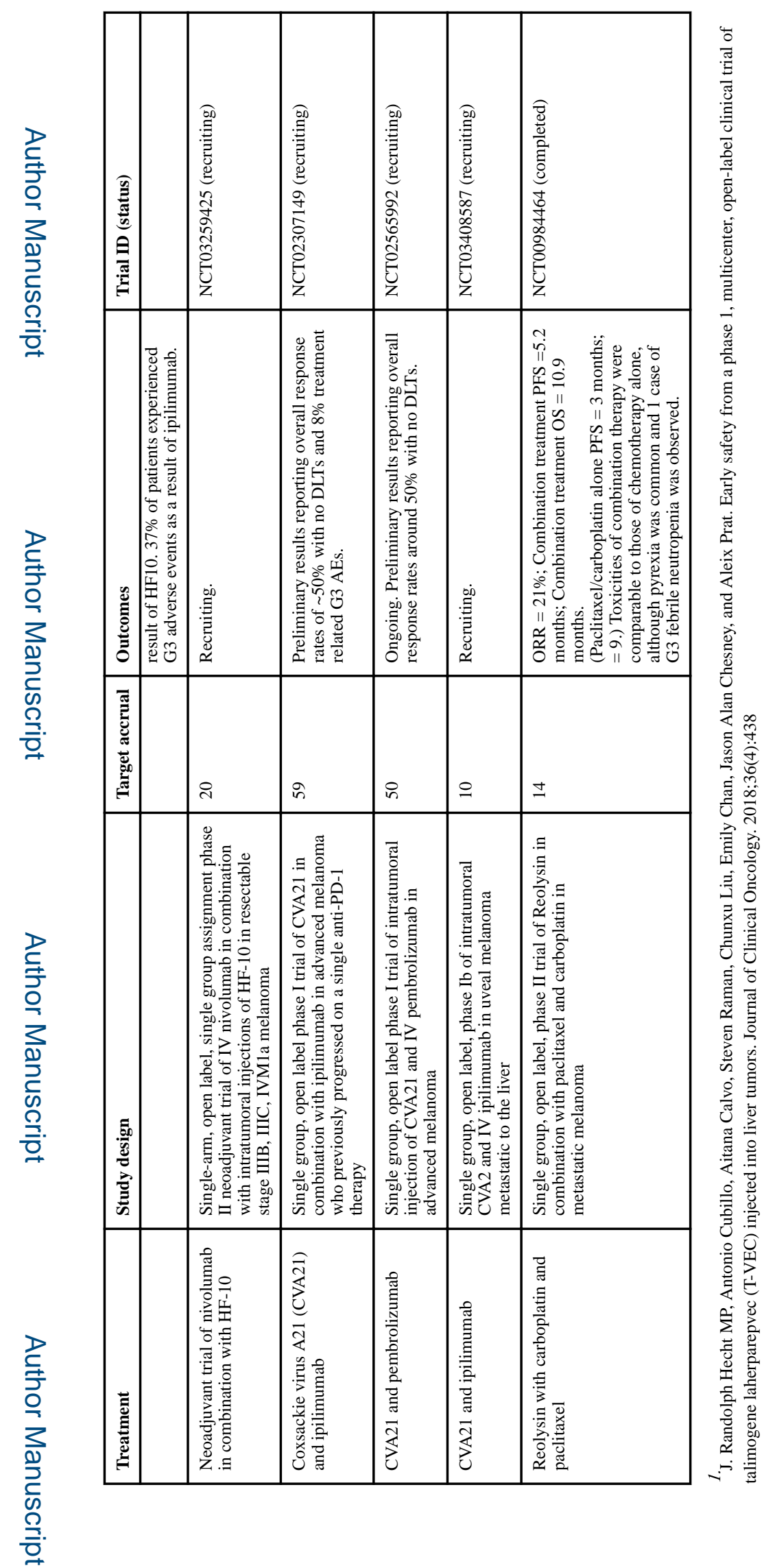

Curr Oncol Rep. Author manuscript; available in PMC 2019 August 25. 